\title{
Investigations of Immune Response and Interleukin-2 Level in Iranian Patients with Common Variable Immunodeficiency
}

\author{
Heidarzadeh Arani Marzieh ${ }^{1}$, Isaeian Ana ${ }^{2}$, Piroozmand Ahmad ${ }^{3 *}$ and Motedayyen Hossein ${ }^{4 *}$ \\ ${ }^{1}$ Department of Pediatrics, Faculty of Medicine, Kashan University of Medical Sciences, Iran \\ ${ }^{2}$ Department of Pathology, Faculty of Medicine, Tehran University of Medical Sciences, Iran \\ ${ }^{3}$ Department of Microbiology, Faculty of Medicine, Kashan University of Medical Sciences, Iran \\ ${ }^{4}$ Autoimmune Diseases Research Center, Kashan University of Medical Sciences, Iran \\ *Corresponding author: Hossein Motedayyen, Autoimmune Diseases Research Center, Kashan University of Medical Sciences, Iran \\ Piroozmand Ahmad, Department of Microbiology, Faculty of Medicine, Kashan University of Medical Sciences Iran
}

\section{ARTICLE INFO}

Received: 幽 December 03, 2019

Published: 彗December 19, 2019

Citation: Heidarzadeh Arani Marzieh, Isaeian Ana, Piroozmand Ahmad, Motedayyen Hossein. Investigations of Immune Response and Interleukin-2 Level in Iranian Patients with Common Variable Immunodeficiency. Biomed J Sci \& Tech Res 24(1)-2019. BJSTR. MS.ID.003993.

Abbreviations: CVID: Common Variable Immunodeficiency; ELISA: Enzyme-Linked Immunosorbent Assay; ESID: European Society for Immunodeficiency; PBMCs: Peripheral Blood Mononuclear Cells; SEM: Standard Error of Mean; PBS: Phosphate Buffered Saline

\section{ABSTRACT}

Common variable immunodeficiency (CVID) is the primary immunodeficiency disease with unknown etiology, characterized by impaired antibody responses. In addition to the defect in humoral immunity, the number and function of $\mathrm{T}$ cells may be impared in patients with CVID. Regarding interleukin-2 (IL-2) is a fundamental role in survival, proliferation, and development of $\mathrm{T}$ cells as the effector arm of cell-mediated immunity, the aim of this study was whether the situation of the cellualr immune system and level of IL-2 in CVID patients differ from healthy subjects. Peripheral blood mononuclear cells were isolated from whole blood of 20 CVID and 10 healthy subjects. The cells were cultured in the presence or absence of phytohemagglutinin $(2.5 \mu \mathrm{l} / \mathrm{ml})$ at $37^{\circ} \mathrm{C}$. The culture supernatant was collected after 72 hours and the IL-2 level was measured by Enzyme-linked immunoabsorbant assay (ELISA).

The clinical and laboratory characteristics of the patients were also studied. CVID patients showed the reduction in the levels of IgG, IgA, and IgM compared to healthy individuals ( $\mathrm{P}<0.0001)$. The CD4+ cell, CD8+ cell, and CD19+ cell frequencies and CD4/CD8+ cell ratio in patients were significantly reduced $(\mathrm{P}<0.001-0.05)$. Stimulated PBMCs of patients significantly produced lower level of IL-2 than healthy controls ( $\mathrm{P}$ $<0.0001$ ). IL-2 level in CVID patients was positively associated with Igm level, CD4 cell frequency, $C D 4 / C D 8$ cell ratio $(P<0.05, R=0.464)$, while this association was negative for CD8+ cell precentage. Based on these findings, IL-2 may be considered as a useful drug supplement to treatment of infectious and non-infectious complications in CVID patient through enhancing cellular immunity.

Keywords: Common Variable Immunodeficiency; Interleukin-2; Cell-Mediated Immunity

\section{Introduction}

Common variable immunodeficiency (CVID) is defined as a heterogenous disease which involves the production of antibodies, espetially immunoglobulin (Ig) types IgG, IgM, and IgA1. Although the etiology of CVID remains unknown, several genetic factors have been proposed for this disorder, including mutations in ICOS,
CD19, BAFF-R, and TACI genes [1]. Patients with CVID mainly suffer from recurrent bacterial infections, autoimmunity, and malignancy, which contribute to a defect in humoral immunity such as the defect in differentiation of B lymphocyte into plasma cell and deficiency in antibody production [2]. Although the defect in 
humoral response is considered as the most important impairment, in some of CVID patients, the reduced number or impaired function of $\mathrm{T}$ lymphocytes have been observed $[3,4]$. Some CVID patients exhibit the reduction in circulating CD4+ T cells, particularly the cells of the CD4+.CD45RA+ subset [5]. Moreover, some patients with CVID indicate a profound defect in the proliferation of antigenstimulated $\mathrm{T}$ cell in vitro [6].

Extensive data from the literature have revealed that several molecular mechanisms may participate in the decreased number and impaired function of $\mathrm{T}$ cells $[4,7,8]$. One of the mechanisms involved in $\mathrm{T}$ cell defects is the reduced production or impaired fuction of cytokins such as IL-2, IL-12, and IL-4 which play the fundamental roles in $\mathrm{T}$ lymphocyte receptor signaling, B cell functions, and correlations between $\mathrm{T}$ and $\mathrm{B}$ lymphocytes $[2,7,9]$. Previous studies have shown that in some cases of CVID patients, $\mathrm{B}$ lymphocytes have an immature phenotype which produce antibody in the presence of factors such as IL-10, IL-4, IL-2, and co-stimulators [8]. On the other hand, $60 \%$ of patients have low secretion of IL-2 upon $\mathrm{T}$ lymphocyte stimulation with mitogens owing to CD4+ $\mathrm{T}$ lymphocyte deficiency, which results from deficiency in bone marrow progenitor cells or defect in thymic maturation of T cells [3]. Regarding the fact that IL-2 has an indispensable role in the development and function of $\mathrm{T}$ cells, the aim of this study was whether the situation of cell-mediated immunity and level of IL-2 produced by stimulated PBMCs from Iranian patients with CVID differ from healthy subjects.

\section{Materials and Methods}

\section{Study Populations}

A total of 20 individuals were recruited among those referred to Allergy and Immunology Department of Children's Medical Center, Kashan, Iran from January 2017 to July 2018 (Table 1). CVID was diagnosed by the specialist according to the European Society for immunodeficiency (ESID) criteria including: hypogammaglobulinemia (with a marked decrease of two out of IgG, IgA, and IgM isotypes at least two standard deviation (SD) below the mean of age and lack of isohemmagglutinin titer), onset of immunodeficiency at greater than two years of age, bacterial recurrent infections, a poor response to vaccination, and the rejection of other causes of humoral immunodeficiencies (hyper-IgM, SIgA, and X-linked agammaglobulinemia) [10,11]. The sampling was performed after 3-4 weeks of the last intravenous immunoglobulin (IVIG, $400 \mathrm{mg} / \mathrm{kg}$ ) injection. None of CVID patients did not receive the drugs which influence the immune system and antibodies production (i.e. gold salts, phenytoin, sulfasalazine, steroids, and antimalarial drugs) at the time of the blood sampling. All of the patients were alive during the study. 10 healthy volunteers without any history of health or immunodeficiency problems were also participated as a control group. This study was approved by the Ethics Committee of Kashan University of Medical Sciences and preformed according to the declaration of Helsinki. All participants gave the informed consent before entering the study.

Table 1: The demographic characteristics of the CVID and healthy subjects.

\begin{tabular}{|c|c|c|}
\hline & CVID Patients & Healthy Control \\
\hline Number & 20 & 10 \\
\hline Age, years (min-max) & $15.95 \pm 11.32(4-55)$ & $19.4 \pm 11.36(8-45)$ \\
\hline Gender (male/female) & $11 / 9$ & $6 / 4$ \\
\hline
\end{tabular}

Age are expressed as mean \pm SD (range).

\section{Peripheral Blood Mononuclear Cells (PBMCs) Isolation}

Heparinized blood samples $(3 \mathrm{ml})$ were obtained from CVID and healthy subjects. PBMCs were isolated using Ficoll density centrifugation according to the manufacturer's instructions (Miltenyi Biotec, Germany). After centrifugation, the cells were collected from the interface between Ficoll and the plasma and washed several times with phosphate buffered saline (PBS). PBMCs were suspended in PBS and cell count was done with a haemocytometer. Cell viability was also measured using trypan blue dye exclusion.

\section{Determination of the Immunologic Situation of Patients}

To evaluate the humoral and cellular immune situations of patients and affirm CVID disease, the serum levels of IgG, IgA, and IgM in CVID patients and healthy volunteers were measured using an enzyme-linked immunoabsorbant assay (ELISA) kit according to the manufacturer's guidlines (Mabtech, Sweden). The serum levels of IgG, IgA, and IgM in healthy individuals served as control group. Furthermore, the expressions of CD3, CD4, and CD19 on PBMCs of patient and healthy subjects were assessed using a FACSCalibur flow cytometry (Becton Dickinson, San Jose, CA). Accordingly, the cells were stained with fluorescein isothiocyanate (FITC) antihuman CD4 (eBioscience, USA), Phycoerythrin/Cyanine5 (PE/ cyn5) anti-human CD19 ( BioLegend, USA), and Phycoerythrin (PE) anti-human CD8 ( BioLegend, USA) antibodies or the matched isotype control IgG for $25 \mathrm{~min}$ at $4^{\circ} \mathrm{C}$. The matched isotype control antibodies were used as negative controls. Afterwards, the cells were washed twice with PBS and the percentages of the stained cells were measured by a FACSCalibur system and then analyzed using FlowJo software (v10.1, FlowJo, Ashland, OR, USA).

\section{PBMC Stimulation and IL-2 Measurement}

The isolated cells were cultured in 96-well, flat-bottomed microtiter plates, at $37^{\circ} \mathrm{C}$ with $5 \% \mathrm{CO}_{2}$. All assays were performed in duplicate. The cells were then stimulated with phytohemagglutinin (PHA, $2.5 \mu \mathrm{l} / \mathrm{ml}$ ). PBMCs cultured without stimulation were considered as negative control. After 72-hour incubation, the supernatant of the cultures were collected and the level of IL-2 was measured using ELISA ( Mab Tech, Sweden) based on the manufacturer's protocol. 


\section{Delayed-Type Hypersensitivity (DTH) Test}

To evaluate the situation of cellular immunity in CVID patients in vivo, DTH test was carried out using purified protein derivative diphtheria, tetanus, and candida albicans antigens. The results of skin test less than $3 \mathrm{~mm}$ for all three antigens were determined as DTH anergy, while the results of $3 \mathrm{~mm}$ or greater for at least one antigen served as DTH positive.

\section{Statistical Analysis}

The results are presented as mean \pm standard error of mean (SEM). Data analysis was done using GraphPad Prism 6 (GraphPad software, San Diego, CA). The comparison of two groups with normal and non-normal distribution were preformed using unpaired t-test and Mann-Whitney test, respectively. Spearman's test was used to determine the correlation coefficients of the data with non-normal distribution and Pearson's test in the case of normal distribution. $\mathrm{P}$ value $<0.05$ was considered statistically significant.

\section{Results}

\section{Description of Patients}

20 CVID subjects (aged $15.95 \pm 11.32$, mean \pm standard deviation, range: 4 to 55 years) were paticipated in the study. The onset of clinical symptoms in patients was varied and occurred from an age of 3 years old to 18 (Table 2). The age in which the illness was diagnosed varied from a range of 2 years old to 19 (data not shown). The most frequent clinical manifestations among CVID patients were respiratory and gastrointestinal infections, bronchectasia, and splenomegaly (Table 2). All CVID patients had respiratory infections, which two had viral infections and one had fungal infections (Table 2). Of the 20 CVID patients, eight were DTH positive, 15 had gastrointestinal infections, ten had bronchectasia, nine had splenomegaly, eight had autoimmunity, five had lymphopenia, four had granulomatous disease (Table 2). Of all CVID patients, three CVID patients had allergy, thrombocytopenia; two had endocrine disease, autoimmune hepatitis, celiac disease, and hemolytic anemia; one had malignancy, myasthenia gravis, colitis ulcerative, and juvenile rheumatoid arthritis (Table 2). Table 2 is depicted the clinical characteristics of CVID subjects.

Table 2: Correlations of IL-2 level with the familial history and clinical characteristics of CVID patients.

\begin{tabular}{|c|c|c|c|c|}
\hline \multicolumn{2}{|l|}{$\begin{array}{l}\text { Clinical/ } \\
\text { Familial History }\end{array}$} & $\begin{array}{c}\text { Frequency } \\
\text { N (\%) }\end{array}$ & Mean IL-2 \pm SD & $P$ value \\
\hline \multirow{2}{*}{ Paternal Relativity } & - & $7(35)$ & $51.71 \pm 103.96$ & \multirow{2}{*}{0.759} \\
\hline & + & $13(65)$ & $38.31 \pm 85.39$ & \\
\hline \multirow{2}{*}{$\begin{array}{l}\text { Family History Imu- } \\
\text { unodeficiency }\end{array}$} & - & $18(90)$ & $47.78 \pm 93.64$ & \multirow{2}{*}{0.49} \\
\hline & + & $2(10)$ & 0 & \\
\hline \multirow{2}{*}{ Admission } & - & $17(85)$ & $50.59 \pm 95.74$ & \multirow{2}{*}{0.383} \\
\hline & + & $3(5)$ & 0 & \\
\hline
\end{tabular}

\begin{tabular}{|c|c|c|c|c|}
\hline \multirow{2}{*}{ Splenomegaly } & - & $11(55)$ & $68.18 \pm 112.66$ & \multirow{2}{*}{0.172} \\
\hline & + & $9(45)$ & $12.22 \pm 36.67$ & \\
\hline \multirow{2}{*}{ Autoimmunity } & - & $12(60)$ & $47.33 \pm 84.21$ & \multirow{2}{*}{0.8} \\
\hline & + & $8(40)$ & $36.5 \pm 103.24$ & \\
\hline \multirow{2}{*}{$\begin{array}{l}\text { DTH positive } \\
\text { DTH anergy }\end{array}$} & & $12(60)$ & $9.17 \pm 31.75$ & \multirow{2}{*}{$0.035^{*}$} \\
\hline & & $8(40)$ & $93.75 \pm 124.06$ & \\
\hline \multirow{2}{*}{ Lymphopenia } & - & $15(75)$ & $57.33 \pm 100.3$ & \multirow{2}{*}{0.225} \\
\hline & + & $5(25)$ & 0 & \\
\hline Respiratory Infection & & $20(100)$ & $43 \pm 89.79$ & - \\
\hline \multirow{2}{*}{ Bronchectasia } & - & $10(50)$ & $56.8 \pm 89.83$ & \multirow{2}{*}{0.507} \\
\hline & + & $10(50)$ & $29.2 \pm 92.34$ & \\
\hline \multirow{2}{*}{$\begin{array}{l}\text { Gastrointestinal } \\
\text { Infection }\end{array}$} & - & $16(80)$ & $36.4 \pm 49.97$ & \multirow{2}{*}{0.855} \\
\hline & + & $4(20)$ & $45.2 \pm 101.03$ & \\
\hline \multirow{2}{*}{ Viral Infection } & - & $17(85)$ & $47.78 \pm 91.66$ & \multirow{2}{*}{0.49} \\
\hline & + & $3(3)$ & 0 & \\
\hline \multirow{2}{*}{ Fungal Infection } & - & $19(95)$ & $45.18 \pm 92.34$ & \multirow{2}{*}{0.636} \\
\hline & + & $1(5)$ & 0 & \\
\hline \multirow{2}{*}{$\begin{array}{c}\text { Granulomatosis } \\
\text { Disease }\end{array}$} & - & $18(90)$ & $53.75 \pm 97.95$ & \multirow{2}{*}{0.296} \\
\hline & + & $2(10)$ & 0 & \\
\hline \multirow{2}{*}{ Allergy } & - & $17(85)$ & $33.41 \pm 73.28$ & \multirow{2}{*}{0.267} \\
\hline & + & $3(3)$ & $97.33 \pm 168.59$ & \\
\hline \multirow{2}{*}{ Malignancy } & - & $19(95)$ & $45.26 \pm 91.66$ & \multirow{2}{*}{0.636} \\
\hline & + & $1(5)$ & 0 & \\
\hline \multirow{2}{*}{ Endocrine Disease } & - & $18(90)$ & $201.00 \pm 128$ & \multirow{2}{*}{0.005} \\
\hline & + & $2(10)$ & $25 \pm 69$ & \\
\hline \multirow{2}{*}{ Other Infections } & - & $9(45)$ & $73.78 \pm 123.32$ & \multirow{2}{*}{0.172} \\
\hline & + & $11(55)$ & $17.82 \pm 40$ & \\
\hline
\end{tabular}

\section{Immunologic Features of CVID Patients}

To determine the situations of humoral and cellular immunity in patients and confirm CVID disease, the serum levels of IgG, IgA, and IgM and frequencies of CD4+, CD8+, and CD19+ cells in CVID patients were assessed. As shown in Figures $1 \mathrm{~A}-\mathrm{C}$, IgG, IgA, and IgM levels in the patients were significantly reduced compared to healthy control ( $\mathrm{P}<0.0001)$. The level of IgG in $50 \%$ of patients was less than $400 \mathrm{mg} / \mathrm{ml}$ (data not shown). The same trend was observed in the percentages of CD4+ and CD19+ cells in CVID patients (Figures 1D, E \& G, P < 0.01-0.05). On the contrary, CD8+ cell frequency was significantly higher in CVID patients than healthy controls (Figures $1 \mathrm{D} \& \mathrm{~F}, \mathrm{P}<0.01$ ). The proportion of CD4+/CD8+ cells revealed that CD4+/CD8+ cell ratio in CVID patients was significantly increased compared to control group (Figure $1 \mathrm{H}, \mathrm{P}<0.0001$ ). 

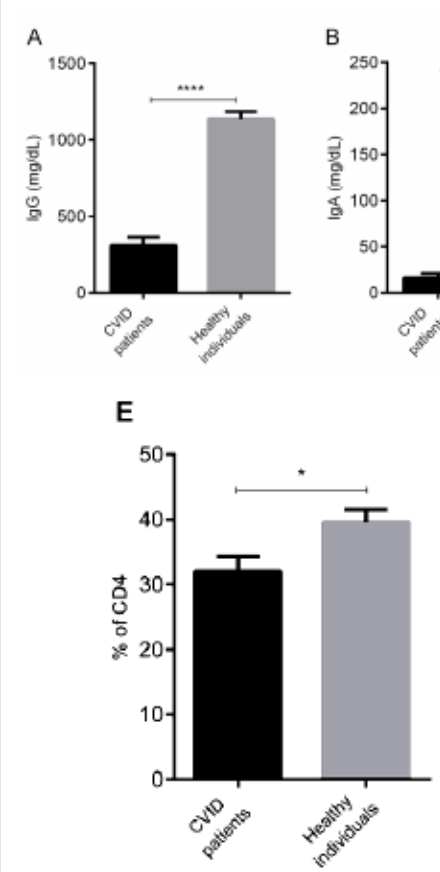

G

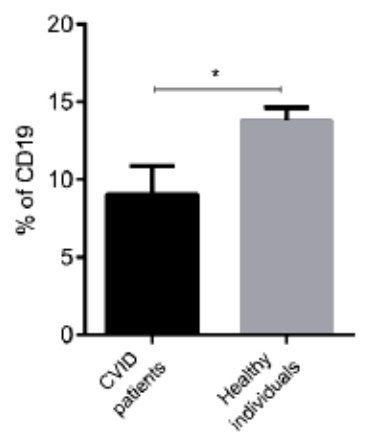

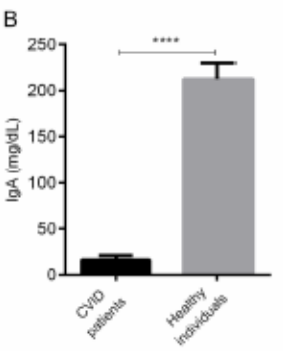

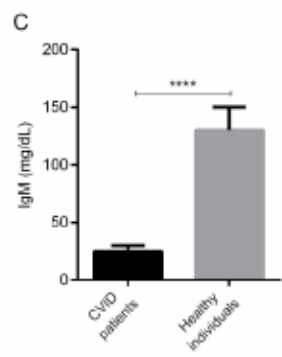

$\mathbf{F}$

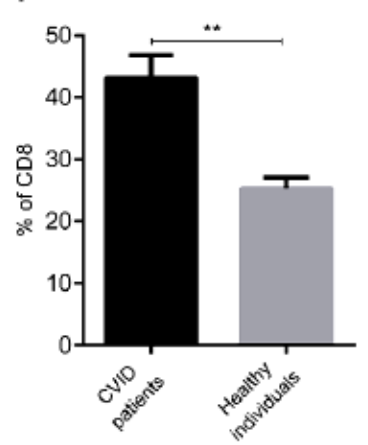

H

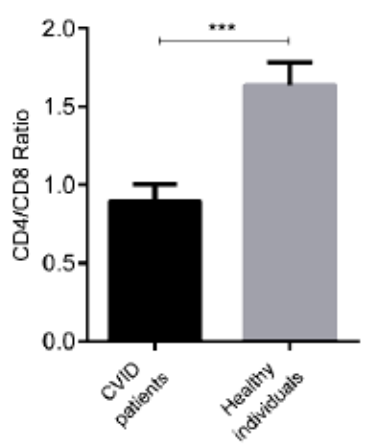

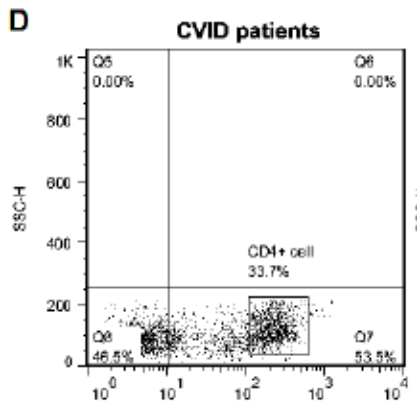
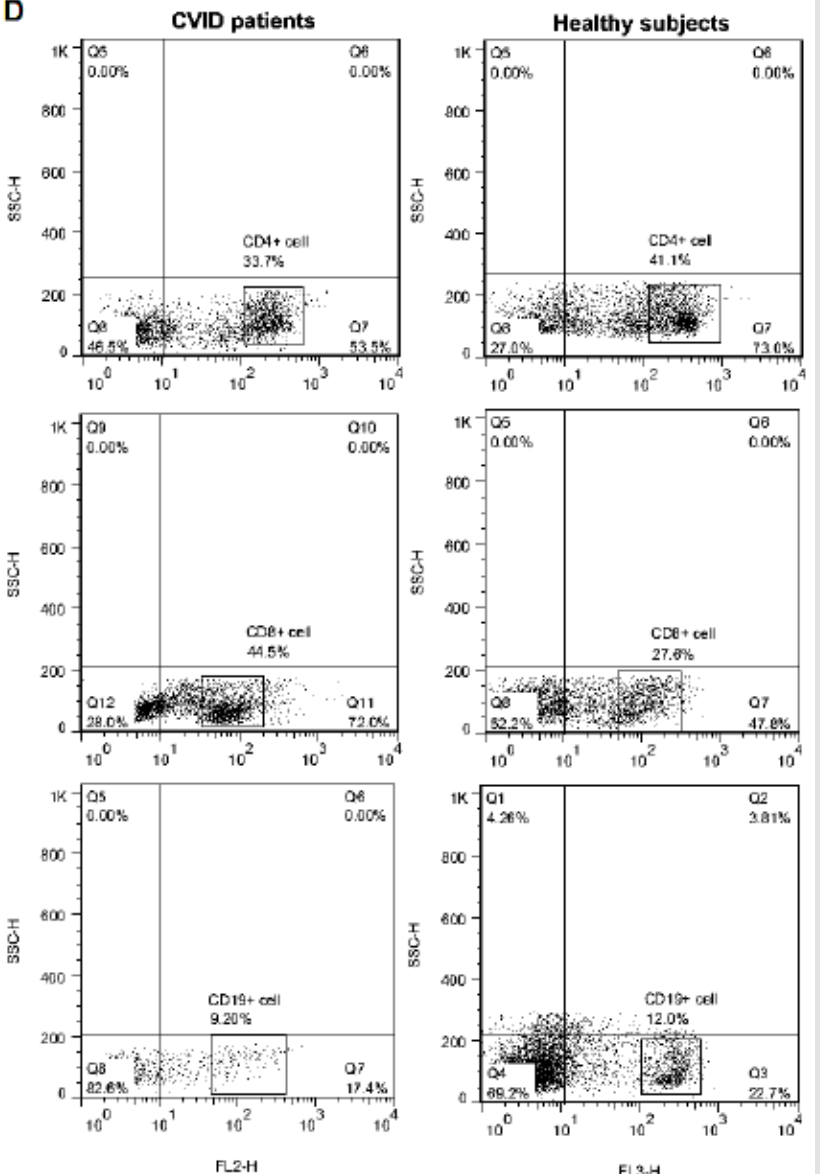

Figure 1: The characteristics of humoral and celluar immunity of CVID and control subjects. The levels of

(A) IgG,

(B) $\operatorname{IgA}$, and

(C) IgM were measured by ELISA. The precentages of CD4+, CD8+, CD19+ and CD4+/CD8+ cell ratio were studied by flow cytomerty (D-H).

The results are representative of 20 independent experiments for CVID group and ten independent experiments for healthy individuals. Each bar in Figure.1 (A-H) shows mean \pm SEM. ${ }^{*} p<0.05,{ }^{* *} p<0.01,{ }^{* * *} p<0.001,{ }^{* * * *} p<0.0001$.

\section{The Assessment of Cytokine Production of PBMCs}

\section{Isolated from CVID Patients}

Regarding the fact that IL-2 participates in key functions of the immune system, especially cell-mediated immunity, IL-2 production of stimulated PBMCs from CVID and healthy subjects was studied. Our results indicated that PBMCs of CVID patients significantly produced lower level of IL-2 than control group (Figure 2, P < 0.0001). Furthermore, IL-2 production in $93.65 \%$ of CVID patients was less than $50 \mathrm{pg} / \mathrm{ml}$ (data not shown).
The levels of IL-2 in Patients with DTH Positive and Anergy

Regarding the fact that IL-2 is an important cytokine in cellmediated immunity, the levels of this cytokine was measured in patients who were DTH positive and anergy, which is an in vivo manifestation of cell-mediated immune response. Our data revealed that patients with DTH positive had significantly higher level of IL-2 than those who had anergy (Figure $3, \mathrm{P}<0.05$ ). The correlation test indicated that IL-2 level in CVID patients was negatively associated with DTH positivity, as expected ( $\mathrm{P}<0.05$, Table 2 ). 


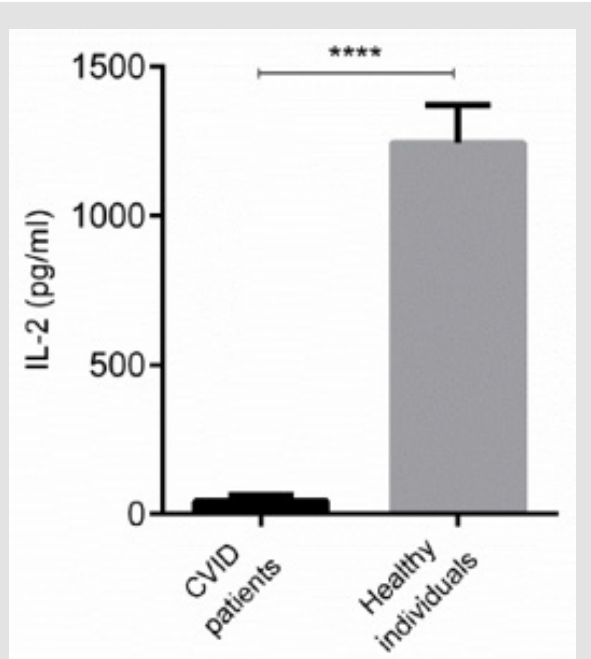

Figure 2: IL-2 levels in CVID and control subjects. The level of IL-2 in CVID and healthy subjects were measured by ELISA. Data are representative of ten independent experiments for healthy subjects and 20 independent experiments for CVID group. Data are shown as mean \pm SEM. ${ }^{* * * *} \mathrm{p}<0.0001$

\section{Correlations of IL-2 Level with Laboratory and Clinical}

\section{Parameters}

The results of Pearson and Spearman tests showed significant positive correlations between CD 4 cell frequency, CD4/CD8 cell ratio and IL-2 level $(\mathrm{P}<0.05, \mathrm{R}=0.464, \mathrm{P}<0.01, \mathrm{R}=0.606$, respectively, Table 3), while the level of IL-2 was negatively associated with

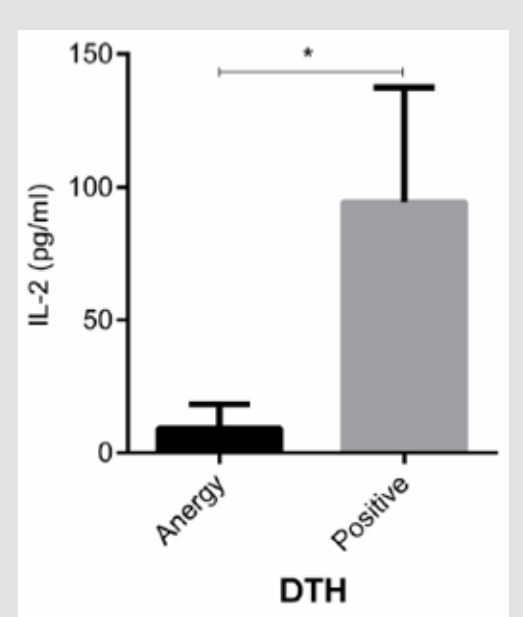

Figure 3: IL-2 levels in CVID patients with DTH positive and anergy. CVID patients were divided into two groups according to DTH positive and anergy. The level of IL-2 in CVID subjects was measured by ELISA. Data are representative of 20 independent experiments for CVID group and ten independent experiments for healthy subjects. Data are shown as mean \pm SEM. ${ }^{*} p<0.05$.

Table 3: Correlations of IL-2 level with the laboratory features of CVID and healthy subjects.

\begin{tabular}{|c|c|c|c|c|}
\hline \multirow{2}{*}{ Laboratory Factors } & \multicolumn{2}{|c|}{ IL-2 in patients } & \multicolumn{2}{c|}{ IL-2 in Healthy Control } \\
\cline { 2 - 5 } & Pearson Correlation & P value & Pearson Correlation & P value \\
\hline CD4 & 0.464 & 0.039 & -0.096 & 0.793 \\
\hline CD8 & -0.453 & 0.045 & 0.209 & 0.562 \\
\hline CD4/CD8 ratio & 0.606 & 0.004 & -0.216 & - \\
\hline CD19 & 0.195 & 0.411 & - & - \\
\hline IgG & 0.355 & 0.125 & - & - \\
\hline IgA & 0.341 & 0.142 & - & 0.749 \\
\hline IgM & 0.516 & 0.02 & -0.104 & \\
\hline
\end{tabular}

\section{Discussion}

CVID is defined as inherent defects in the immune system which results in susceptibility to recurrent bacterial infections, due to hypogammaglobulinemia and impaired antibody responses [12]. Moreover, several non-infectious complications have been reported for CVID patients, including cancer, autoimmunity, and splenomegaly [1]. Although large proportion of CVID patients suffer from the lack of immunoglobulin production by B cells, some patients experience the reduced number and impaired function of $\mathrm{T}$ lymphocytes [4]. The reduction in T-cell cytokines has a pathogenic
CD8+ cell percentage $(\mathrm{P}<0.05, \mathrm{R}=-0.453$, Table 3$)$. Other results indicated that age and gender of patients did not effect on IL-2 level. In contrast with healthy control, the level of IL-2 was significantly associated with IgM level in CVID patients $(\mathrm{P}<0.05, \mathrm{R}=0.516$, Table 3). Among clinical factors, there was a negative correlation between the IL-2 level and prevalence of endocrine disease ( $\mathrm{P}<$ $0.005, \mathrm{R}=0.520$, Table 3).

role in some cases of the CVID patients. We therefore investigated whether IL-2, as an important cytokine in cellular-mediated immunity, participate in the reduced number and defected function of T cells in CVID patients. In the present study, the levels of IgG, IgM, and IgA were significantly lower in CVID patients than healthy subjects. In agreement with these findings, previous studies have been indicated that CVID patients faild to produce the normal level of IgG, IgM, and IgA compared to healthy subjects [13,14]. On the other hand, our data revealed that CD19+ cell frequency in the patients was significantly reduced. These findings demonstrate that 
why patients with CVID suffer from various infections. It is needless to say that defective antibody production plays an indispensable role in susceptibility to frequent respiratory infections and poor response to vaccination and pathogenic agents [1].

Regarding the fact that CD4+ T helper cells play a fundamental role in the production of antibodies by B cells, the features of cellular immunity of CVID patients were also studied. In consistent with the results of antibody productions, percentage of CD4+ cells, unlike CD8+ cells, in patients was significantly reduced compared to healthy subjects, which are consistent with other reports showing a decrease in naive CD4+ T cells and an oligoclonal expansion of CD8+ T cells in the patients $[15,16]$. In line with this notion, Viallard et al. demonstrated that CD8+DR+ T-lymphocyte percentage was significantly increased in MB0 classified patients, characterized by fewer CD27+ B cells [17]. As expected, the results of the porportion of $\mathrm{CD} 4+/ \mathrm{CD} 8+$ cells revealed that this ratio was notably reduced in CVID patients. These observations suggest that patients with CVID had a defect in CD4+ helper T cells, which may participate in the impairment of antibody productions. Having considered that T-cell cytokines are essential to differentiation, proliferation, and development of $\mathrm{T}$ cells, the critical question was whether the reduced numbers of CD4+ cells contribute to IL-2 defect. The production of IL-2 of the stimulated PBMCs of CVID patients was studied. We found that the cells of patients with CVID failed to produce IL-2 following stimulation with PHA.

This finding is consistent with many studies pointing to CD4+ and CD8+ T cells from CVID patients produce significantly lower level of IL-2 upon stimulation via T cell receptor and costimulation $[8,18]$. In an effort to further confirm the possible role of IL-2 in impaired cellular immunity of CVID patients, the level of IL-2 in patients with DTH positive and anergy was determined. The results indicated that IL-2 level in patients with DTH positive differed from those who had anergy. CVID patients with DTH positive showed higher level of IL-2 than patients with DTH anergy. Therefore, it is likely that reduction in CD4+ cell numbers may result in the decreased production of IL-2 in CVID patients. In the next step, the correlations of IL-2 level with CD4+ and CD8+ cell frequencies were evaluated. Interestingly, our data demonstrated that IL-2 level was positively associated with CD4+ cells, while this association was negative for CD8+ cells. Moreover, there is a significantly positive correlation between IL-2 level and IgM level in CVID patients. These results may provide another evidence to indicate that the reduced IL-2 participate in the defect of cell-meidate immunity, thereby lead to reduction of antibody productions in patients with CVID.

\section{Conclusion}

The results of this study, in consistent with previous studies, provide additional evidence to show that IL-2 defect contributes to impaired cellular immunity in Iranian patients with CVID, thereby may result in defective humoral immunity. This finding suggests that IL-2 may be considered as a useful drug supplement to treatment of infectious and non-infectious complications in CVID patient through improving cell-mediated immunity.

\section{Acknowledgement}

We appreciate all people who helped us in this study, especially the personnel of Pediatric Medical Center and Research Center of Asthma and Allergy. This work was financially supported by Kashan University of Medical Sciences (KAUMS).

\section{Conflict of Interest}

The authors report no conflict of interest.

\section{References}

1. Sadati ZA, Motedayyen H, Sherkat R, Ostadi V, Eskandari N ( 2019) Comparison of the Percentage of Regulatory $\mathrm{T}$ cells and their p-STAT5 Expression in Allergic and Non-Allergic Common Variable Immunodeficiency Patients. Immunol Invest 48(1): 52-63.

2. Buckley RH (2002) Primary immunodeficiency diseases: dissectors of the immune system. Immunol Rev 185: 206-219.

3. Kondratenko I, Amlot P, Webster A, Farrant J (1997) Lack of specific antibody response in common variable immunodeficiency (CVID) associated with failure in production of antigen-specific memory T cells. Clin Experi Immunol 108(1): 9-13.

4. Farrington M, Grosmaire LS, Nonoyama S, Fischer SH, Hollenbaugh D, et al. (1994) CD40 ligand expression is defective in a subset of patients with common variable immunodeficiency. Proc Nat Academy Sci 91(3): 1099-1103.

5. Farrant J, Spickett G, Matamoros N, Copas D, Hernandez M, et al. (1994) Study of B and $\mathrm{T}$ cell phenotypes in blood from patients with common variable immunodeficiency (CVID). Immunodeficiency 5(2): 159-169.

6. Stagg A, Funauchi M, Knight S, Webster A, Farrant J (1994) Failure in antigen responses by $\mathrm{T}$ cells from patients with common variable immunodeficiency (CVID). Clin Experi Immunol 96 (1): 48-53.

7. Di Renzo M, Zhou Z, George I, Becker K, Cunningham Rundles C (2000) Enhanced apoptosis of $\mathrm{T}$ cells in common variable immunodeficiency (CVID): role of defective CD28 co-stimulation. Clin Experi Immunol 120 (3): 503-511.

8. Ten R, Anderson P, Zein N, Temesgen Z, Clawson ML, et al. (2002) Interleukin-2 liposomes for primary immune deficiency using the aerosol route. Int Immunopharmacol 2(2-3): 333-344.

9. Eisenstein EM, Jaffe JS, Strober W (1993) Reduced interleukin-2 (IL-2) production in common variable immunodeficiency is due to a primary abnormality of CD4+ T cell differentiation. J Clin Immunol. 13(4): 247258.

10. Ameratunga $\mathrm{R}$, Brewerton $\mathrm{M}$, Slade $\mathrm{C}$, Jordan A, Gillis $\mathrm{D}$, et al. (2014) Comparison of diagnostic criteria for common variable immunodeficiency disorder. Front Immunol 5: 415.

11. Ameratunga R, Woon ST, Gillis D, Koopmans W, Steele R (2013) New diagnostic criteria for common variable immune deficiency (CVID), which may assist with decisions to treat with intravenous or subcutaneous immunoglobulin. Clin Exp Immunol 174(2): 203-211.

12. Aghamohammadi A, Farhoudi A, Moin M, Rezaei N, Kouhi A, et al. (2005) Clinical and immunological features of 65 Iranian patients with common variable immunodeficiency. Clin Diagn Lab Immunol 12(7): 825-832.

13. Mellemkjaer L, Hammarström L, Andersen V, Yuen J, Heilmann C, et al. (2002) Cancer risk among patients with IgA deficiency or common variable immunodeficiency and their relatives: a combined Danish and Swedish study. Clin Exp Immunol 130(3): 495-500. 
14. Quinti I, Soresina A, Spadaro G, Martino S, Donnanno S, et al. (2007) Long-term follow-up and outcome of a large cohort of patients with common variable immunodeficiency. J clin Immunol 27(3): 308-316.

15. Mouillot G, Carmagnat M, Gérard L, Garnier JL, Fieschi C, et al. (2010) $\mathrm{B}$-cell and T-cell phenotypes in CVID patients correlate with the clinical phenotype of the disease. J Clin Immunol 30(5): 746-755.

16. Serrano D, Becker K, Cunningham Rundles C, Mayer L (2000) Characterization of the $\mathrm{T}$ cell receptor repertoire in patients with common variable immunodeficiency: oligoclonal expansion of CD8+ T cells. Clin Immunol 97(3): 248-258.

ISSN: 2574-1241

DOI: 10.26717/BJSTR.2019.24.003993

Piroozmand A, Hossein M. Biomed J Sci \& Tech Res

(C) This work is licensed under Creative Commons Attribution 4.0 License

Submission Link: https://biomedres.us/submit-manuscript.php
17. Viallard JF, Blanco P, André M, Etienne G, Liferman F, et al. (2006) CD8+ HLA-DR+ T lymphocytes are increased in common variable immunodeficiency patients with impaired memory B-cell differentiation. Clin Immunol 119(1): 51-58.

18. Thon V, Wolf H, Sasgary M, Litzman J, Samstag A, et al. (1997) Defective integration of activating signals derived from the T cell receptor (TCR) and costimulatory molecules in both CD4+ and CD8+ T lymphocytes of common variable immunodeficiency (CVID) patients. Clin Exp Immunol 110(2): 174-181.

$\begin{array}{ll}\text { BIOMEDICAL } & \text { Assets of Publishing with us } \\ \text { RESEARCHES } & \text { - Global archiving of articles } \\ \text { - Immediate, unrestricted online access } \\ \end{array}$

